\title{
Anaplasmose beim Hund - Infektion häufig, Krankheit selten
}

\author{
Michèle Bergmann, Katrin Hartmann
}

Anaplasma-phagocytophilum-Infektionen sind in Deutschland endemisch. Viele Hunde sind mit Anaplasmen infiziert, entwickeln aber keine klinischen Symptome. Gerade in letzter Zeit gibt es immer mehr Fallberichte von nachgewiesener klinisch manifester Anaplasmose. Bei rechtzeitiger Diagnosestellung ist die Behandlung unproblematisch.

\section{Åtiologie}

Der Erreger Anaplasma (A.) phagocytophilum ist in Deutschland endemisch und kann die sogenannte canine granulozytäre Anaplasmose verursachen [6]. Die Übertragung auf den Hund erfolgt meist über Schildzecken (Ixodes spp.) [4]. Hierfür muss die Zecke 36-48 Stunden am Wirt anhaften [12], [13]. Infektionen finden vor allem in den Frühjahrs-, Sommer- und Herbstmonaten statt, in denen die Aktivität der Zecken ihren Höhepunkt erreicht. Es werden vor allem Hunde infiziert, die sich viel im Freien aufhalten [1], [4], [8], [16]. Die Infektion wird durch verschiedene genetische Varianten des Erregers hervorgerufen, die vermutlich die Pathogenität der Erregerstämme beeinflussen [4].

In Deutschland haben $1 / 3-1 / 5$ aller Hunde Antikörper gegen A.phagocytophilum [15]. Sie haben sich infiziert, aber nur wenige von ihnen entwickeln eine Krankheit. Eine erhöhte Anfälligkeit für Infektionen mit A. phagocytophilum bei bestimmten Rassen oder verschiedenen Altersgruppen wurde in deutschen Hundepopulationen nicht nachgewiesen. Allerdings scheinen männliche Hunde häufiger infiziert zu sein als weibliche [1].

A.platys, der Erreger der caninen zyklischen Thrombozytopenie, ist in Deutschland nicht endemisch. Infektionen bei

\section{Anaplasmen}

Anaplasmen sind obligat intrazelluläre, gramnegative Bakterien aus der Familie der Anaplasmataceae (Gattung:

Anaplasma). Zusammen mit den Ehrlichien gehören sie zu den Rickettsien (Ordnung: Rickettsiales). Die beim Hund in Deutschland am häufigsten diagnostizierte Anaplasmen-Gattung ist Anaplasma (A.) phagocytophilum. Unter dieser Bezeichnung werden die folgenden früher einzeln benannten Erreger zusammengefasst [6]:

- Ehrlichia equi

- Ehrlichia phagocytophila

- humane-granulozytäre-EhrlichioseAgens (HGE-Agens)

Hunden sind in Deutschland daher sehr selten und kommen in der Regel nur vor, wenn die Tiere im mediterranen Ausland waren [2].

\section{Pathogenese}

Etwa 4-7 Tage nach einer Infektion mit A.phagocytophilum kommt es zu einer Bakteriämie. Die Dauer der Bakteriämie bei natürlicher Infektion ist noch nicht eindeutig bekannt. In einigen Studien wird ein Zeitraum von ungefähr 4 Wochen angegeben. Im Blut infiziert A.phagocytophilum vor allem neutrophile Granulozyten. Von dort aus gelangt der Erreger in den gesamten Körper und infiziert verschiedene Organe wie Leber, Lunge, Nieren und zentrales Nervensystem (ZNS). Die meisten infizierten Hunde haben allerdings trotz Bakteriämie keine klinischen Symptome [4]. Es ist nicht genau bekannt, ob eine A.-phagocytophilum-Infektion wieder eliminiert werden kann. Vermutlich ist ein einmal infizierter Hund lebenslang infiziert und auch eine
Therapie führt nicht zur Erregerelimination.

\section{Klinische Symptome}

Nur sehr wenige Hunde mit A.-phagocytophilum-Infektion werden krank. Der weitaus überwiegende Teil aller natürlich mit A.phagocytophilum infizierten Hunde erkrankt nie. Gerade in letzter Zeit gibt es jedoch immer mehr Beschreibungen von Hunden mit klinisch manifester Anaplasmose. Warum ein Hund erkrankt und die meisten anderen nicht, ist nach wie vor unklar. Der Schweregrad der Erkrankung kann von mild und selbstlimitierend bis hin zu einer schweren Verlaufsform variieren.

In den beschriebenen symptomatischen Fällen nach natürlicher Infektion tritt die Krankheit sehr akut nach der Infektion auf. Die Infektion führt zu einer meist fieberhaften Allgemeinerkrankung. Wie bei allen Rickettsien-Infektionen hat eine Infektion mit A. phagocytophilum bei klinischer Manifestation eine Thrombozytopenie zur Folge. Daher sind die häufigsten Symptome:

- Fieber

- petechiale Blutungen

- Epistaxis

Ebenso kann es durch die Thrombozytopenie zu Blutungen in verschiedenen Organen kommen. Weiterhin beschrieben sind Symptome des Bewegungsapparats wie Bewegungsunlust und Lahmheiten durch Polyarthritis sowie blasse Schleimhäute durch Anämie.

Durch A.phagocytophilum ausgelöste Entzündungsreaktionen in verschiedenen Organen, wie Lunge, Niere, Leber und Milz, können in selteneren Fällen zu Husten, Polydipsie, gastrointestinalen Symptomen sowie zu Spleno- und Lymphadeno- 


\section{Experimentelle Infektion}

Werden Hunde experimentell mit A. phagocytophilum infiziert, sind die vorherrschenden klinischen Symptome einer Anaplasmose ein reduziertes Allgemeinbefinden, generalisierte Lymphadenopathie und Fieber. Die Schwere der Symptomatik variiert bei unterschiedlichen A.-phagocytophilumStämmen. Labordiagnostisch zeigten alle experimentell infizierten Hunde eine Thrombozytopenie und Leukopenie [17].

Nach experimenteller Infektion können auch chronische Infektionen entstehen, bei denen A. phagocytophilum in verschiedenen Organen wie Knochenmark, Milz und Leber persistiert. Diese chronisch infizierten Hunde haben jedoch keine Symptome. Eine Reaktivierung der Infektion mit dem erneuten Auftreten von Symptomen kann allerdings mehrere Monate später auftreten. Diese Reaktivierung kann z. B. durch die Gabe von immunsuppressiven Medikamenten ausgelöst werden. Die Reaktivierung kann Symptome wie eine erhöhte Körpertemperatur und labordiagnostische Veränderungen, z. B. Thrombozytopenie und Lymphozytose, zur Folge haben [9].

megalie führen. Neurologische Symptome wie Anfälle, Ataxie und propriozeptive Defizite sind ebenfalls beschrieben und entstehen vermutlich durch Blutungen in das ZNS oder Entzündungsreaktionen, die zu Gewebeschäden im ZNS führen [4], [7], [8], [11], [14], [16].

\section{Laboruntersuchungen}

Labordiagnostisch haben Hunde mit klinisch manifester Anaplasmose immer eine Thrombozytopenie. Die Ursache der Thrombozytopenie ist multifaktoriell und oft sekundär immunmediiert. In solchen Fällen können antithrombozytäre Antikörper nachgewiesen werden. Der Erreger infiziert vor allem neutrophile Granulozyten und Zellen des mononukleären Phagozytosesystems (Milz, Leber und Knochenmark). Nach einer Infektion kann es zur Zerstörung infizierter Zellen kommen. Mögliche weitere Laborveränderungen sind daher:

- Neutropenie

- aregenerative Anämie

- erhöhte Aktivitäten der Leberenzyme

Oft tritt eine Hyperglobulinämie und gleichzeitig eine reaktive Hypalbuminämie auf. Selten kommt es zu einer Hyperbilirubinämie und regenerativen Anämie durch eine sekundär immun-mediierte Hämolyse [4], [7], [8], [9], [14], [16].

\section{Diagnose}

Eine Anaplasmose kann nicht allein anhand von klinischen Symptomen diagnostiziert werden. Diese sind unspezifisch und viele andere Krankheiten kommen differenzialdiagnostisch in Betracht. Ein Erregernachweis ist daher unbedingt erforderlich.

\section{Direkter Erregernachweis}

\section{Mikroskopische Untersuchung}

Der Nachweis von Anaplasmen ist mit verschiedenen Methoden möglich. Mit einer mikroskopischen Untersuchung von Blutausstrichen können granulozytäre Einschlusskörperchen (Morulae) in neutrophilen Granulozyten im akuten Stadium der Infektion nachgewiesen werden. Diese beweisen das Vorhandensein von Rickettsien-artigen Erregern, lassen aber keine Unterscheidung der Spezies Anaplasma und Ehrlichia zu [4]. Außerdem geht eine Anaplasmose nicht zwingend mit sichtbaren intragranulozytären Morulae einher [7].

\section{.konkret}

Eine mikroskopische Untersuchung ist nur im positiven Fall beweisend.

\section{PCR}

Die Polymerasekettenreaktion (PCR) ist sensitiver als der Nachweis von Morulae in neutrophilen Granulozyten. Mittels PCR kann die DNA von A.phagocytophilum im Blut nur in frühen Stadien der Infektion nachgewiesen werden. Bei experimentell infizierten Hunden konnte der Erreger somit ab dem 2. Tag nach der Infektion über einen Zeitraum von 20-60 Tagen gefunden werden [9], [17]. Die PCR wird also negativ, obwohl die Infektion persistiert. Sie ist daher zum Nachweis einer akuten A.-phagocytophilum-Infektion geeignet [11].

Da die klinisch manifeste Anaplasmose meist sehr akut auftritt, ist eine positive PCR sogar Voraussetzung für die Diagnose. Allerdings sind viele Hunde mit positiver PCR nicht erkrankt.

\section{.konkret}

Eine positive PCR allein ist nicht zum Nachweis der Krankheit ausreichend.

\section{Indirekter Erregernachweis}

Ein indirekter Erregernachweis (Nachweis von Antikörpern gegen Anaplasmen im Serum) ist mittels Immunfluoreszenz (IF) oder Enzyme-Linked Immunosorbent Assay (ELISA) möglich. Mittlerweile ist auch ein kommerzieller Schnelltest (SNAP 4Dx assay/SNAP 3Dx assay, IDEXX Laboratories, Inc., Westbrook, ME) für die Praxis erhältlich [2].

Der Nachweis von Antikörpern ist im frühen Infektionsverlauf (bis zu 30 Tage nach der Infektion mit A.phagocytophilum) negativ, da in diesem Zeitraum häufig noch keine Antikörper vorhanden sind [5], [7]. Hunde, bei denen Antikörper gegen $A$. phagocytophilum nachgewiesen werden, haben eher eine chronische Infektion. Das Vorkommen von Antikörpern bei Hunden lässt somit keine Rückschlüsse auf die klinische Manifestation einer Infektion zu. Da Hunde, wenn sie nach einer Infektion mit $A$. phagocytophilum überhaupt erkranken, ganz akut Symptome zeigen, ist bei diesen Hunden der Antikörpernachweis in der Regel noch negativ.

\section{.konkret}

Der Antikörpernachweis eignet sich nicht zur Diagnose einer akuten,

klinisch manifesten Anaplasmose.

\section{Bestätigung der Diagnose}

Die Diagnose der Krankheit Anaplasmose ist also nicht ganz unproblematisch. Weder der direkte noch der indirekte Erregernachweis lassen Rückschlüsse auf das Vorliegen einer klinisch manifesten 


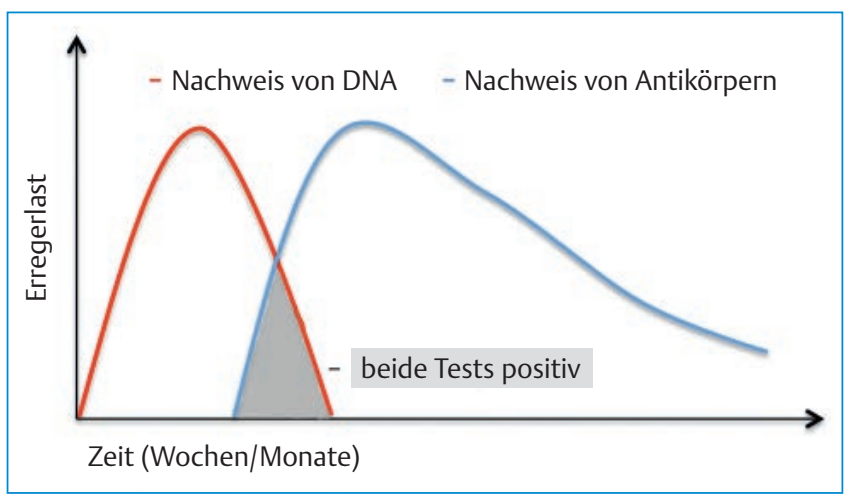

Abb. 1 Verlauf des direkten Erregernachweises (DNA mittels PCR) und des indirekten Erregernachweises (Antikörper) bei Hunden mit A.-phagocytophilum-Infektion (nach Eberts et al. [7], Scorpio et al. [17]). @ M. Bergmann u.

K. Hartmann

Anaplasmose zu. Da bis zu 1/3 aller Hunde in Deutschland infiziert sind, aber keine Symptome haben, erlaubt ein Nachweis des Erregers bei einem Hund mit Krankheitssymptomen nicht automatisch die Diagnose Anaplasmose. Die Diagnose klinisch manifeste Anaplasmose kann daher nur gestellt werden, wenn eine Befundkombination gegeben ist.

Für die Diagnosestellung müssen 3 Befunde vorliegen:

1. positiver direkter Erregernachweis (positive A.-phagocytophilum-PCR oder Morulae im Blutausstrich)

2. Vorhandensein einer Thrombozytopenie

3. Anstieg der Thrombozytenzahl im Therapieverlauf mit Doxycyclin innerhalb von wenigen Tagen

Der Nachweis von Antikörpern fällt bei Hunden mit akuter Anaplasmose meist noch negativ aus ( $\checkmark$ Abb. 1$)$. Ob chronische, klinisch manifeste Verlaufsformen überhaupt existieren, ist unklar. Sie wären schwierig zu diagnostizieren. Genauso schwierig ist der Nachweis einer reaktivierten Anaplasmose, da unklar ist, ob der direkte Erregernachweis in solchen Fällen wieder positiv wird.

\section{Therapie und Prognose}

Die Therapie einer Anaplasmose-Infektion sollte nur bei Hunden mit Symptomen erfolgen. Hunde, bei denen eine akute Anaplasmose erkannt und behandelt wird, haben in der Regel eine gute Prognose. Die Therapie der Wahl besteht aus der Gabe von Doxycyclin ( $5 \mathrm{mg} / \mathrm{kg}$ p.o.) alle 12 Stunden über 3 Wochen. Die meisten Hunde zeigen nach der Gabe von Doxycyclin ein schnelles Ansprechen auf die Therapie. Ein Ansprechen auf die Therapie ist definiert als Anstieg der Thrombozytenzahl. Die regelmäßige Kontrolle der Thrombozytenzahl ist daher als Therapiemonitoring geeignet. Etwa $3 / 4$ aller Hunde zeigen bereits innerhalb von 1-2 Tagen nach Therapiebeginn eine Besserung. Das restliche $1 / 4$ zeigt spätestens 6 Tage nach Therapiebeginn eine Besserung [4], [16].

Nach erfolgreicher Behandlung von Hunden mit experimenteller A.-phagocytophilum-Infektion dauerte es 7-8 Monate, bis 
keine Antikörper im Serum mehr nachweisbar waren [8]. Bei natürlich infizierten Hunden konnten in einigen Fällen noch 12 Monate nach der Behandlung Antikörper gegen A. phagocytophilum nachgewiesen werden [16].

\section{Prophylaxe}

Eine Impfung gegen $A$. phagocytophilum gibt es in Deutschland nicht. Die Kontrolle der Zecken mithilfe von Spot-onPräparaten oder Halsbändern mit repellierendem Effekt ist die wichtigste Maßnahme zur Prävention einer Infektion. Ein Zeckenschutz sollte ganzjährig erfolgen. Das frühzeitige Absammeln der Zecken am Hund reduziert das Risiko einer Übertragung der Anaplasmen [12], [13].

Da eine Übertragung der Anaplasmen auch durch Transfusion von Blut infizierter Hunde erfolgen kann, sollte bei allen Spenderhunden vor der
Blutspende eine A.-phagocytophilumPCR durchgeführt werden [10].

\section{Bedeutung als Zoonose}

Eine Infektion mit Anaplasmen kann sowohl bei Menschen als auch bei Tieren auftreten. Die Übertragung der Anaplasmen beim Menschen erfolgt wie beim Hund hauptsächlich über Zecken. Eine Übertragung des Erregers vom Hund auf den Menschen wurde bislang nicht beschrieben. Direkte Infektionen durch Hunde spielen höchstwahrscheinlich keine Rolle. Allerdings stellt der Kontakt mit dem Blut infizierter Wildtiere ein gewisses Infektionsrisiko für den Menschen dar [6], [19]. Dies sollte auch beim Umgang mit Blut von A.-phagocytophiluminfizierten Hunden beachtet werden.

Online zu finden unter http://dx.doi.org/10.1055/s-0035-1558509

\section{Literatur}

1 Barth C, Straubinger RK, Sauter-Louis C et al. Prevalence of antibodies against Borrelia burgdorferi sensu lato and Anaplasma phagocytophilum and their clinical relevance in dogs in Munich, Germany. Berl Munch Tierarztl Wochenschr 2012; 125 (7-8): 337-344

2 Barth C, Straubinger RK, Müller E et al. Comparison of different diagnostic tools for the detection of Anaplasma phagocytophilum in dogs. Vet Clin Pathol 2014; 43 (2): 180-184

3 Cardoso L, Tuna J, Vieira L et al. Molecular detection of Anaplasma platys and Ehrlichia canis in dogs from the North of Portugal. Vet J 2010; 183 (2): 232-233

4 Carrade DD, Foley JE, Borjesson DL et al. Canine granulocytic anaplasmosis: a review. J Vet Intern Med 2009; 23 (6): 1129-1141

5 Chandrashekar R, Mainville CA, Beall MJ et al. Performance of a commercially available in-clinic ELISA for the detection of antibodies against Anaplasma phagocytophilum, Ehrlichia canis, and Borrelia burgdorferi and Dirofilaria immitis antigen in dogs. Am J Vet Res 2010; 71 (12): 1443-1450 
6 Dumler JS, Barbet AF, Bekker CP et al. Reorganization of genera in the families Rickettsiaceae and Anaplasmataceae in the order Rickettsiales: unification of some species of Ehrlichia with Anaplasma, Cowdria with Ehrlichia and Ehrlichia with Neorickettsia, descriptions of six new species combinations and designation of Ehrlichia equi and 'HGE agent' as subjective synonyms of Ehrlichia phagocytophila. Int J Syst Evol Microbiol 2001; 51 (Pt 6): 2145-2165

7 Eberts MD, Vissotto de Paiva Diniz PP, Beall $\mathrm{MJ}$ et al. Typical and atypical manifestations of Anaplasma phagocytophilum infection in dogs. J Am Anim Hosp Assoc 2011; 47 (6): 86-94

8 Egenvall AE, Hedhammar AA, Bjöersdorff Al. Clinical features and serology of 14 dogs affected by granulocytic ehrlichiosis in Sweden. Vet Rec 1997; 140 (9): 222-226

9 Egenvall A, Lilliehöök I, Bjöersdorff A et al. Detection of granulocytic Ehrlichia species DNA by PCR in persistently infected dogs. Vet Rec 2000; 146 (7): 186-190

10 Ewing SA, Dawson JE, Panciera RJ et al. Dogs infected with a human granulocytotropic Ehrlichia spp. (Rickettsiales: Ehrlichieae). J Med Entomol 1997; 34 (6): 710-718

11 Granick JL, Armstrong PJ, Bender JB. Anaplasma phagocytophilum infection in dogs: 34 cases (2000-2007). J Am Vet Med Assoc 2009; 234 (12): 1559-1565

12 Hodzic E, Fish D, Maretzki CM et al. Acquisition and transmission of the agent of human granulocytic ehrlichiosis by Ixodes scapularis ticks. J Clin Microbiol 1998; 36 (12): 35743578

13 Katavolos P, Armstrong PM, Dawson JE et al. Duration of tick attachment required for transmission of granulocytic ehrlichiosis. J Infect Dis 1998; 177 (5): 1422-1425

14 Kohn B, Galke D, Beelitz P et al. Clinical features of canine granulocytic anaplasmosis in 18 naturally infected dogs. J Vet Intern Med 2008; 22 (6): 1289-1295

15 Krupka I, Pantchev N, Lorentzen L et al. Durch Zecken übertragbare bakterielle Infektionen bei Hunden: Seroprävalenzen von Anaplasma phagocytophilum, Borrelia burgdorferi sensu lato und Ehrlichia canis in Deutschland. Prakt Tierarzt 2007; 88: 776788

16 Poitout FM, Shinozaki JK, Stockwell PJ et al. Genetic variants of Anaplasma phagocytophilum infecting dogs in Western Washington State. J Clin Microbiol 2005; 43 (2): 796801
17 Scorpio DG, Dumler JS, Barat NC et al. Comparative strain analysis of Anaplasma phagocytophilum infection and clinical outcomes in a canine model of granulocytic anaplasmosis. Vector Borne Zoonotic Dis 2011; 11 (3): 223-229

18 Suksawat J, Hegarty BC, Breitschwerdt EB. Seroprevalence of Ehrlichia canis, Ehrlichia equi, and Ehrlichia risticii in sick dogs from North Carolina and Virginia. J Vet Intern Med 2000; 14 (1): 50-55

19 Telford SR. Risk for acquiring human granulocytic ehrlichiosis: exposure to deer blood or deer ticks? Clin Infect Dis 1997; 24 (3): 531533

\section{Dr. Michèle Bergmann}

Medizinische Kleintierklinik, LMU

Veterinärstr. 13

80539 München

n.bergmann@medizinische-kleintierklinik.de

\section{Prof. Dr. Katrin Hartmann}

Medizinische Kleintierklinik, LMU

Veterinärstr. 13

80539 München

vorstandsassistenz@medizinische-

kleintierklinik.de 